\title{
Fragrance Formation in Aquilaria spp. Shoot Culture Induced by Acremonium sp.
}

\author{
GAYUH RAHAYU \\ Biology Department, Faculty Mathematics and Natural Sciences, Institut Pertanian Bogor, Campus Darmaga, \\ Bogor 16680, Indonesia.
}

\begin{abstract}
In this research, fragrance formation in the shoot culture of three clones of Aquilaria malaccensis (Ama1, Ama7 and Ama13) and four clones of A. microcarpa (Ami5, Ami8, Ami16 and P6) as a response towards infection of four isolates of Acremonium sp. (F, G, L, and M) were studied using dual culture methods in three different concentrations of a modified Murashige-Skoog (MSmod) media (50, 75 and $100 \%$ ). The result indicated that Acremonium $\mathrm{F}$ and $\mathrm{M}$ induced the formation of fragrance. The index of fragrance induced by Acremonium $\mathrm{F}$ was twice from that of Acremonium $\mathrm{M}$, and commonly produced in shoot cultures in MSmod $50 \%$. The index of fragrance in A. malaccensis shoot culture was $70 \%$ higher than that of $A$. microcarpa. Furthermore, GLC analyses of acetone extracted from fragranced shoots of $A$. malaccensis indicated that only one compound (RT 5.76) might determine the fragrance. Host-microbe compatibility study indicated that the presence of Acremonium significantly affected the fitness of the shoots. In contrast, the presence of shoots did not significantly affect the growth rate of Acremonium. In general, Acremonium $\mathrm{F}, \mathrm{L}$ and $\mathrm{M}$ significantly increased shoot death during the 35 days of interaction.
\end{abstract}

Key words: Fragrance, Aquilaria, Acremonium, fungal growth response, percentage of dead shoots, fragrance index

Agarwood is produced in trees of Aquilaria and Gyrinops species. In Indonesia, agarwood is mainly harvested from A. malaccensis, A. microcarpa, A. filaria and Gyrinops verstegii. Agarwood is an accumulation of resinaceous substances produced by the tree and deposited in the heartwood. The resin is produced as a part of the tree defense reaction towards mechanical damage or fungal infection. Acremonium $\mathrm{F}$ has been proved to be a potential agent to induce agar formation in young trees of Aquilaria crassna, A. malaccensis and A. microcarpa (Rahayu et al. 1999). It took more than 4 months of incubation for fragrance formation in these trees.

A preliminary study on the dual culture of shoot cultures of Aquilaria and Acremonium (Rahayu et al. 2001) indicated that this method can be utilized to evaluate the capability of agents, both the fungi and the plant hosts, in fragrance formation. About 20 clones of Aquilaria have been maintained as shoot cultures, yet the potential of these clones to produce agar substance has not been known. The same thing happened to the Acremonium isolated from agarwood. The objective of this study is, therefore, to evaluate the response (in terms of fragrance formation) of three clones of A. malaccensis (Ama1, Ama7 and Ama13) and four clones of A. microcarpa (Ami5, Ami8, Ami16 and P6) towards four Acremonium isolates (F, G, L, M) infection, in three concentrations of modified Murashige Skoog medium (MSmod 100\%, 75\% and 50\%).

\section{MATERIALS AND METHODS}

Source of Explants and Shoot Cultures. The stocks of shoot cultures of three clones of $A$. malaccensis (Ama1, Ama 7and Ama 13) and four clones of A. microcarpa (Ami5, Ami8, Ami16 and P6) of the collection of Biotechnology and Tree Breeding Laboratory, BIOTROP, were used as sources of explants. The shoots were first multiplied by cutting one or two nodes, and the nodes were planted in shoot inducing

*Corresponding author: Phone/Fax: +62-0251-8622833; E-mail: gayuhrahayu@yahoo.com media for 2 months. The Murashige Skoog modified media (MSmod) with BAP $1 \mathrm{mg} \mathrm{L}^{-1}$ (Situmorang 2000) was used as inducing media. The new formed shoots were then elongated for 1 month in MSmod. All cultures were maintained at $26^{\circ} \mathrm{C}$ with $16 \mathrm{~h}$ photoperiods of cool-light of 80 lux. The elongated shoots were served as sources of shoots in dual culture.

Fungal Isolates and Source of Inoculum. The stocks of culture of four Acremonium sp. isolates (F, G, L, M) of the collection of the Mycology Laboratory, Bogor Agricultural University were used. These isolates were first regenerated in potato sucrose agar in Petri dishes and incubated at room condition. A one-week-old Acremonium culture was used as a source of inoculum in a dual culture.

Fragrance Formation. Fragrance formation was studied in a dual culture of shoots of a clone of Aquilaria sp and an Acremonium isolate. The dual culture was made in three concentrations of MSmod, i.e. $100 \%, 75 \%$ and $50 \%$. Firstly, two or three vigorous shoots of Aquilaria sp. were planted in MSmod about $2 \mathrm{~cm}$ from the edge of the Petri dishes. A small inoculum $(0.5 \mathrm{~cm}$ diameter $)$ of each culture of Acremonium sp. was then planted at a distance of $4.5 \mathrm{~cm}$ from the shoots. All treatments were done in 3 replicates. A single culture of either Acremonium or shoots was used as control. Prior to determination of fragrance formation, the response of Acremonium towards the presence of shoots and the fitness of the shoots in the presence of Acremonium were observed. The data obtained were analyzed using univariate analyses of SPSS version 13. The Duncan's multiple range test was used to compare means of treatments. A significant difference was determined at $\alpha 0.05$.

The response of Acremonium towards the presence of the shoots was stated as the colony radial growth rate (RGR) of the Acremonium (Peters et al. 1998). The colony growth was observed every two days and stopped when the colony was in contact with the shoots. The response of the shoots was observed weekly until all shoots died or at a maximum of 60 days of interaction and calculated as percentage of dead shoots (PDS). 
The fragrance formation was measured by the organoleptic method and followed by the gas liquid chromatography (GLC) analyses of the fragrant shoots. The organoleptic determination was done by five persons whom have experience in agarwood fragrance and stated as fragrant indexes (FI: 0, no fragrance produced; 1, slightly produced fragrance; 2, moderately produced fragrance; 3 , strongly produced fragrance).

Agar Substance Extraction and Its GLC Analyses. When the interaction ended, the fragrance shoots and control shoots were ground in a mortar to make paste. The paste was then extracted in $15 \mathrm{~mL}$ of acetone and blended with a magnetic stirrer. The extract was filtrated through Whatman no 1 filter paper which was sprinkled with $\mathrm{Na}_{2} \mathrm{SO}_{4}$ anhydrous. The filtrate was concentrated until $1 / 10$ of its volume. The components of agar substances were determined qualitatively using GLC with Carbowax packed column 20 on Chromosorb WAW 80/100 mesh, injector temperature of $23^{\circ} \mathrm{C}$, oven column temperature of $195^{\circ} \mathrm{C}$ and $\mathrm{N}_{2}$ carrier gas $(50 \mathrm{~mL} / \mathrm{min})$, air velocity $\left(0.55 \mathrm{~kg} / \mathrm{cm}^{2}\right)$ and an FID detector. Retention time (Rt) of the components of agar substance from the extracted shoots was compared with that of the natural agarwood. Peaks with similar Rt were considered as similar components of agar substance.

\section{RESULTS}

Fragrance Formation. The fragrance produced (stated as FI) depended on the isolates of Acremonium, clones of Aquilaria and MSmod concentrations. Acremonium $F$ induced fragrance with the highest index (1.73), followed by Acremonium M (0.83). Furthermore, Acremonium G (0.07) and $\mathrm{L}(0)$ were considered not inducing fragrance formation (Fig 1). In general, A. malaccensis produced fragrance with index higher than $A$. microcarpa. Amongst the $A$. malaccensis clones, Ama7 produced the highest grade and was significantly different from the two other clones (Fig 2), whilst the fragrance produced by all clones of $A$. microcarpa varied and were significantly different (Fig 2). The FI was also determined by the compatibility of Acremonium and the clones. Acremonium $\mathrm{F}$ and $\mathrm{M}$ might induce fragrance production in all clones, while Acremonium G induced fragrance only in Ama1 and Ama7.

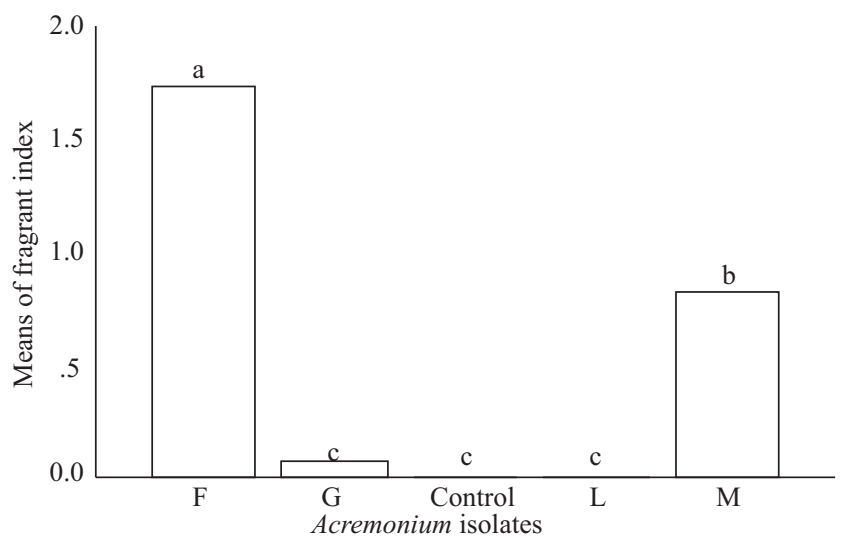

Fig 1 Capability of Acremonium isolates to induce fragrance [stated as Fragrance Index (FI). Bar with different letters are significantly different by Duncan test at $\alpha 0.05]$.

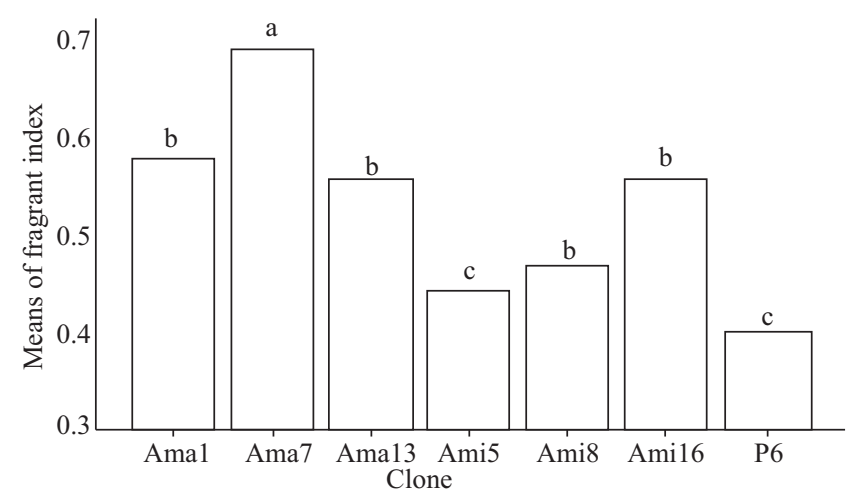

Fig 2 Fragrance produced in various clones of Aquilaria. Bar with different letters are significantly different by Duncan test at $\alpha 0.05$.

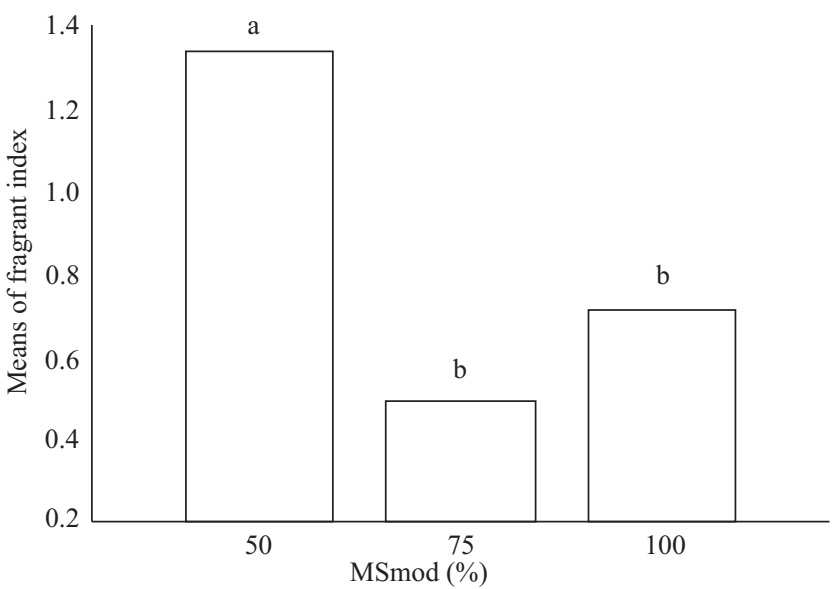

Fig 3 Fragrance induced by Acremonium $\mathrm{F}$ in shoot planted in various Msmod concentration. Bar with different letters are significantly different by Duncan test at $\alpha 0.05$.

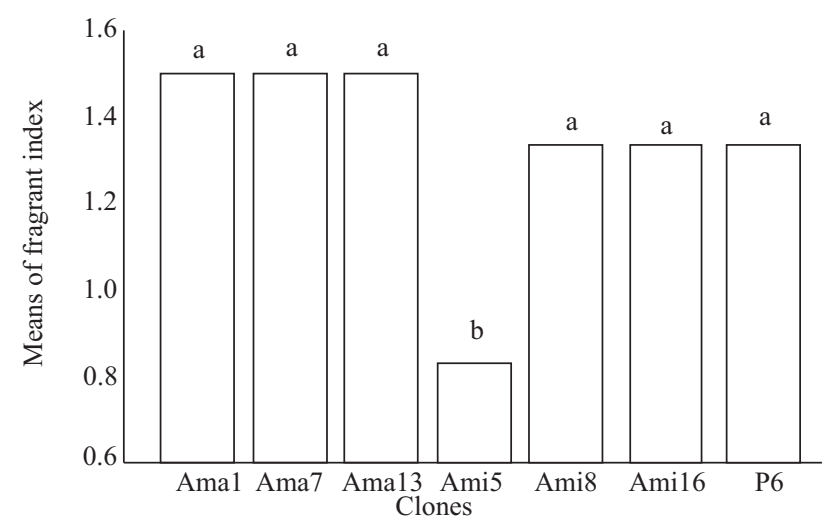

Fig 4 Fragrance produced by various clones as induced by Acremonium F. Bar with different letters are significantly different by Duncan test at $\alpha 0.05$.

Shoots planted in different MSmod concentrations showed FI in significant differences. The shoots planted in MSmod 50\% produced the highest FI (1.18), followed by those planted in MSmod 100\% (0.65) and MSmod 75\% (0.47). This indicated that nutrition limitation enhanced plant stress.

Interaction of the three factors was also observed. As it is too complicated to explain, the result reported here is strictly 
on the role of Acremonium $\mathrm{F}$ in inducing fragrance formation in various clones at various MSmod concentrations. Fragrance grades as induced by Acremonium $\mathrm{F}$ in all clones are insignificantly different. However, the optimum MSmod concentration for Acremonium to induce fragrance is 50\% (Fig 3). In this media, all but Ami5 produced an insignificant different FI (Fig 4).

The colony growth was observed for about 3 weeks. In the third week some of the colonies had been in contact with the shoots. During fungal-plant interaction, the colony diameter was neither inhibited nor stimulated (Fig 1). Acremonium grew further covering the shoots.

Statistic analyses indicated that the RGR of the colonies of all Acremonium isolates were not significantly affected by the presence of the shoots, but by the presence of the media concentration. The RGR of the colonies of Acremonium in MSmod $100 \%$ and $50 \%$ were greater and were significantly different from those of $75 \%$ (Table 1). Furthermore, the growth of Acremonium $\mathrm{G}$ was the slowest and significantly different from the other Acremonium isolates (Table 1). The optimal media concentration for each Acremonium isolate varied. Acremonium $\mathrm{F}$ and $\mathrm{G}$ grew best on MSmod $50 \%$, but Acremonium L on MSmod 100\% (Table 1).

Table 1 Radial growth rate* of Acremonium in various concentrations of Msmod

\begin{tabular}{clcrc}
\hline \multirow{2}{*}{$\begin{array}{c}\text { Concentration of } \\
\text { MSmod (\%) }\end{array}$} & \multicolumn{4}{c}{ Acremonium } \\
\cline { 2 - 5 } & $\mathrm{F}$ & $\mathrm{G}$ & $\mathrm{L}$ & $\mathrm{M}$ \\
\hline 50 & $1.89^{\mathrm{a}}$ & $1.26^{\mathrm{b}}$ & $1.25^{\mathrm{b}}$ & $1.53^{\mathrm{a}}$ \\
75 & $1.16^{\mathrm{b}}$ & $0.06^{\mathrm{b}}$ & $1.25^{\mathrm{b}}$ & $1.47^{\mathrm{a}}$ \\
100 & $1.35^{\mathrm{a}}$ & $0.07^{\mathrm{b}}$ & $1.93^{\mathrm{a}}$ & $1.89^{\mathrm{a}}$ \\
\hline
\end{tabular}

*Stated as rate of radial growth colony increment ( $\mathrm{mm} /$ day).

Data followed by different letters are significantly different by Duncan test at $\alpha 0.05$.

Shoots started to show browning leaves when in contact with the fungus. However, Acremonium L might cause browning leaves prior to contact. Browning leaves usually indicated that the shoots started to die. As a preliminary observation, shoots with brown leaves never recovered. They were considered as dead shoots. Liquid drops were sometimes formed on the surface of the yellowing (Fig 5) or browning leaves.

The PDS was affected significantly in the presence of certain Acremonium isolates and MSmod concentrations. Acremonium M caused the highest PDS (88.89\%), followed by $\mathrm{F}(69.44 \%), \mathrm{L}(57.54 \%)$ and $\mathrm{G}(23.02 \%)$, respectively (Fig 6). In MSmod 100\% and 50\%, the PDS was greater and significantly different from that of $75 \%$ (Fig 7). The PDS was also affected by the interaction between Acremonium and MSmod concentrations. Acremonium F caused the highest PDS in dual cultures with shoots planted either in MSmod $50 \%$ or MSmod $100 \%$. This PDS was not significantly different from the PDS of shoots in dual cultures with M planted in the same concentration as Msmod.

All clones, except for those planted in dual cultures with Acremonium F, were not significantly different in their PDS.
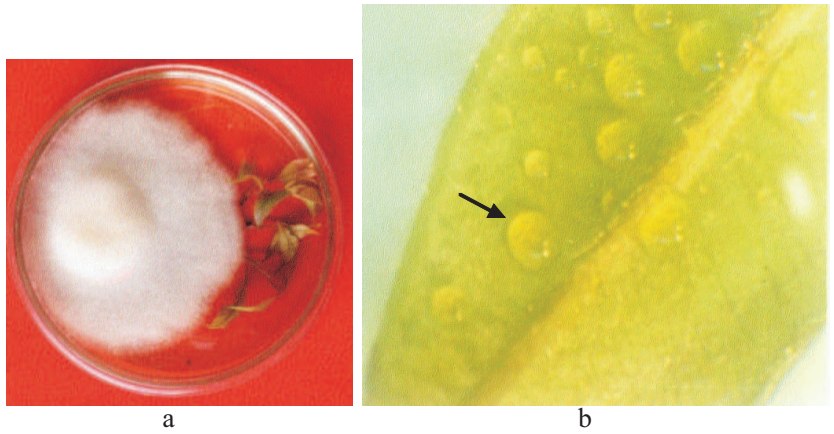

Fig 5 Dual culture of Acremonium $\mathrm{F}$ and Aquilaria malaccensis in 20 days of incubation (a), and liquid drops formed on the lower surface of the leaf of shoot in dual culture (b).

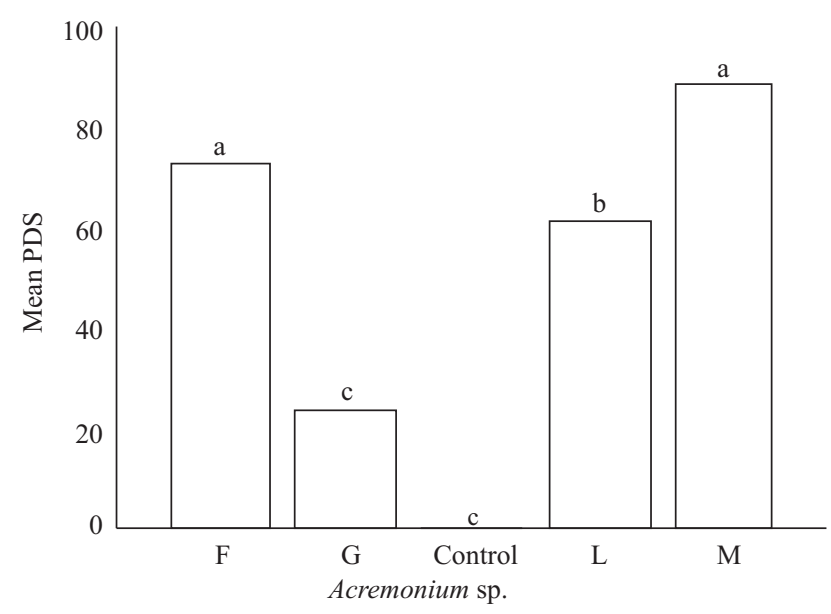

Fig 6 The percentage of death shoot (PDS) caused by various Acremonium isolates. Bar with different letters are significantly different by Duncan test at $\alpha 0.05$.

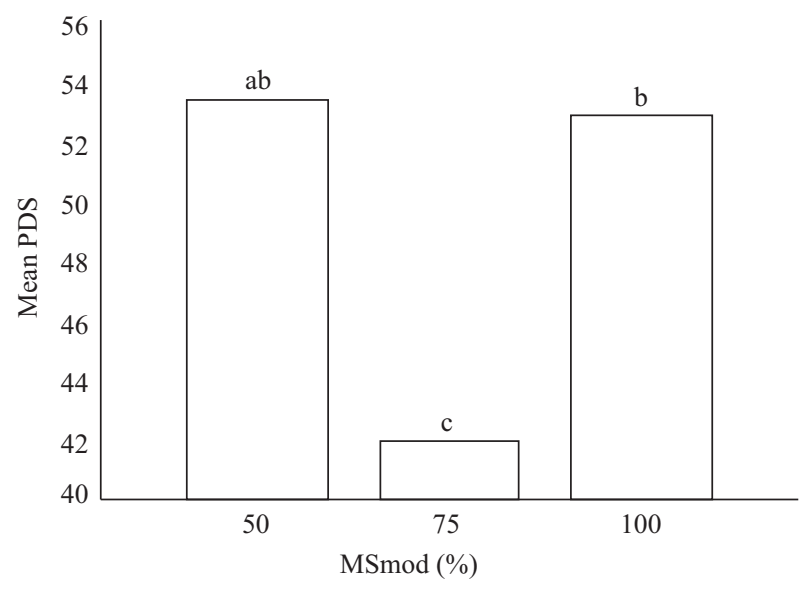

Fig 7 The percentage of death shoot (PDS) in various MSmod concentrations. Bar with different letters are significantly different by Duncan test at $\alpha 0.05$.

A. microcarpa $\mathrm{P} 6$ was the most resistant clone, in contrast to Ami 8, which was the most sensitive clone (Fig 8). The PDS of clones also varied significantly when planted in MS mod 50\%. A. microcarpa Ami 5 was the fittest clone (Fig 9).

GLC Analyses. Fourteen components of agarwood substance were detected from natural agarwood. However, the fragrance shoots contained a maximum of 8 components. By comparing to the agarwood substance in shoots which were planted in single cultures, three new components were 


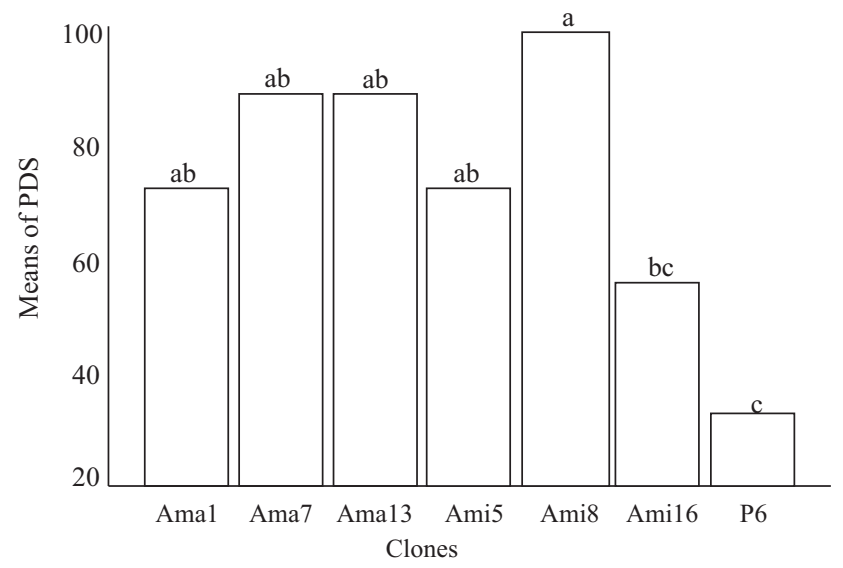

Fig 8 The percentage of death shoot (PDS) of various clones caused by Acremonium F. Bar with different letters are significantly different by Duncan test at 0.05 .

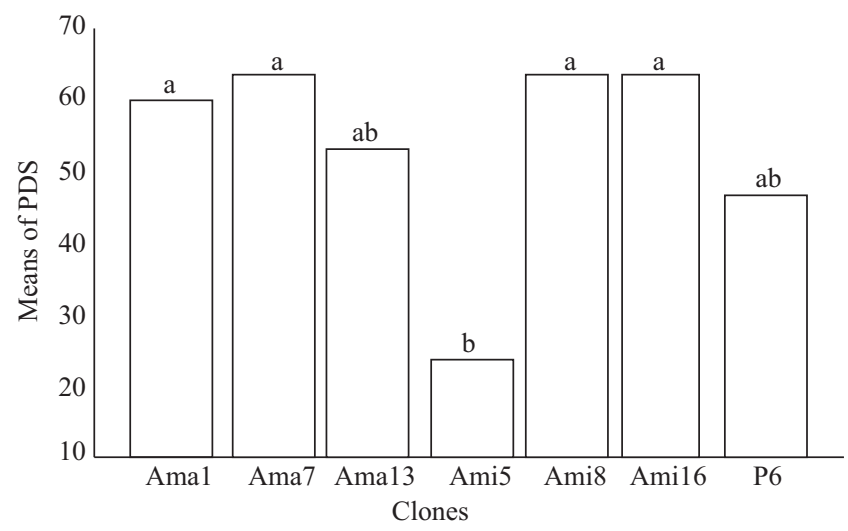

Fig 9 The percentage of death shoot (PDS) of various clones planted in MSmod $50 \%$. Bar with different letters are significantly different by Duncan test at $\alpha 0.05$.

produced in shoots planted in the dual cultures. These are the substances with Rt 5.76, 22.27, and 27.46. Of those substances, the component with Rt 5.76 was the most frequently formed in dual cultures. This was then considered as the substances that produced fragrance.

\section{DISCUSSION}

Degree of Fragrance. A compatible interaction between Acremonium and agarwood clones produces specific agarwood fragrance. Acremonium $\mathrm{F}$ and $\mathrm{M}$ showed a wider compatibility in terms of host species ranges rather than did Acremonium $\mathrm{G}$ and L. Although the fragrance produced among host species or within species was significantly different, the capability of individual shoots to give response to infection should also be counted. Age and Hill (1994) asserted that the response given by the tall fescue due to Acremonium infection varied depending on the plant genotype. There are genotypes with high concentration of ergovaline and those with low concentration of ergovaline.

Not all shoots were capable of producing fragrance. The mechanism of response is not clear yet. In the controlled condition, as in the dual culture, the amount of nutrition available might be critical in producing fragrance. Shoots that grew in half the strength of MSmod medium were capable of producing fragrance stronger than those that grew in full strength of MSmod medium. This indicated that when the plant was stressed, more fragrance was produced. According to Ramawat and Sonie (1999), secondary metabolite was produced when plant was understress. Therefore, the degree of fragrance might not be a specific reaction to infection, but rather a response to all kinds of stress.

Although it has been stated that nutrition can be a critical factor for fragrance formation, the media for shoot cultures are generally suitable for Acremonium growth. On the basis of their RGR, it is known that the behavior of two Acremonium isolates, i.e. $\mathrm{F}$ and $\mathrm{G}$, is different from the others. Isolates $\mathrm{F}$ and $\mathrm{G}$ grew best at $50 \%$ while the other grew best at $100 \%$. The differences in behavior toward nutrition may be due to the differences in the sources of isolates. Acremonium $\mathrm{F}$ was obtained from G.verstegii (Lombok), whereas the $\mathrm{G}$ and $\mathrm{L}$ isolates were from $A$. malaccensis (Riau), and $\mathrm{M}$ was from unknown agarwood (Papua). Character differences among Acremonium isolates had been reported by Carson (1999), who reported isolates of Acremonium from Cucurbitaceae. She mentioned that these Acremonium might differ in their production of chlamydospores. Some isolates showed prolific production of chlamydospores, while others produced only a small number. Rahayu (2001) also reported that Acremonium from agarwood could be differentiated on the basis of their sensitivity to benomil, but their sensitivity to benomil did not depict their RGR in Msmod.

The RGR of Acremonium isolates were not influenced by the presence of the shoots. It might be no host secretion that affected the fungal growth. This is in contrast to Sieber et al. (1990), who stated that the growth of Cryptodiaphorthe hytrix was strongly stimulated by the presence of host callus Acer macrophyllum. Water-soluble metabolites produced by the callus may be responsible for this stimulation. Similarly, Lu and Clay (1994) stated that Atkinsonella grew faster on their native hosts than on alien hosts. The growth of the Atkinsonella isolated from Danthonea sericea was stimulated by $D$. sericea calli, whereas the $D$. spicata isolate was inhibited on the third day of growth relative to the controls. Furthermore, it was stated that the dual culture represents an in vitro system for screening compatibility of host-pathogen, in this case clavicipitaceous fungi with both native and alien hosts, according to Peters et al. (1998)

The presence of shoots did not affect the RGR of Acremonium. However, the presence of Acremonium significantly reduced the health of the shoots. The shoots were dead after being in contact with Acremonium, except for those that were paired with Acremonium. This indicated that Acremonium may affect shoot health in a different way.

To initiate an infection, germ-tubes of these Acremonium do not produce appresorium. Therefore, the infection may not be due to a mechanical process. According to Pitson et al. (1997), Acremonium and Cephalosporium produced $\beta$ glucanase and $\beta$-glucosidase that might destroy the polysaccharides of the plant cell walls. These enzymes were inducible. Therefore, these enzymes would only be 
produced by the fungus when the fungus was in contact with the plant. This might explain why the vigorousness of the shoots was reduced after being in contact with the fungus. Furthermore, Cephalosporium graminearum was reported to produce graminin which might cause the wilting of the leaves of Triticum aestivum (Rahman et al. 2001). Further research on the ability of these isolates to produce certain types of enzymes and toxins should be conducted.

The pathogenicity of different isolates also varied. Like other phytopathogenic fungi, this phenomenon is quite common. Although $\mathrm{M}$ is the most pathogenic isolate, the fragrance produced by the dying shoots is lower than that by Acremonium F. The resistance of the Aquilarian clones towards Acremonium $\mathrm{F}$ also varied and was not correlated with the fragrance produced.

GLC Analyses. Fourteen components of agarwood substance were detected from natural agarwood. According to Yuan (1995), about 30 sesquiterpenoids compound have been detected in natural agarwood originated from various Aquilaria spp. Among those components, oxo-agarospirol was considered as the components responsible for the fragrance. In the shoots of the dual cultures, only one component with Rt 5.76 out of 8 components detected was considered as the substances that produced fragrance. This compound has not yet identified.

\section{ACKNOWLEDGEMENT}

This study was made possible by a grant given by the Competitive Research Grant XI, of the Directorate of Higher Education, Ministry of National Education. The authors are grateful to Jonner Situmorang, Ana Sepriana, Titik Kartika, and Arif Nuryadin for their laboratory assistance.

\section{REFERENCES}

Age CS, Hill NS. 1994. Ergovaline variability in Acremonium infected tall fescue due to environment and plant genotype. Crop Sci 34:221-6.

Lu M, Clay K. 1994. Differential growth of Atkinsonella species on host calli. Mycologia 86: 667-73.

Peters S, Draeger S, Aust HJ, Schulz B. 1998. Interactions in dual culture of endophytic fungi with host and non-host calli. Mycologia 90:360-7.

Pitson SM, Seviour RJ, McDaugall BM. 1997. Production of $\beta$-glucan degrading enzymes by Acremonium and Cephalosporium spesies. Mycol Res 101:153-8.

Rahayu G, Isnaini Y, Situmorang J. 2001. Characteristics Acremonium isolates from agar symptom in agarwood tree: morphology, isozymes pattern and their sensitivity [in Indonesian]. Proceeding of national congress XVI and scientific seminar of Indonesian Association of Phytopathology; 2001 Aug 22-24; Bogor, Indonesia. Bogor: PFI. p 455-67.

Rahayu G, Isnaini Y, Umboh MIJ. 1999. Induction potency of some hyphomycetes for agar formation in sapling of agarwood tree [in Indonesian]. Proceeding of national congress XV and scientific seminar of Indonesian Association of Phytopathology; 1999 Sep 16-18; Purwokerto, Indonesia. Purwokerto: PFI. p 573-81.

Rahman M, Mundt CC, Wolpert TJ, Riera-Lizarazu O. 2001. Sensitivity of wheat genotypes to a toxic fraction produced by Cephalosporium graminearum and correlation with disease susceptibility. Phytopathology 91:702-7.

Ramawat KG, Sonie KC. 1999. Production under stress. In Ramawat KG, Merilon JM, (eds). Biotechnology on secondary metabolites. Enfield, New Hampshire: Science Publ.

Sieber NS, Sieber-Canavesi F, Dorworth CE. 1990. Simultaneous stimulation of endophytic Cryptodiaporthe hystrix and inhibition of Acer macrophyllum callus in dual culture. Mycologia 82:560-75.

Situmorang J. 2000. Micropropagation of agarwood tree (Aquilaria sp.) from Riau and identification of their genetic traits [in Indonesian]. [thesis]. Bogor: Institut Pertanian Bogor.

Yuan, QS. 1995. Aquilaria species: in vitro culture and the production of eaglewood (agarwood). In: Bajaj YPS, editor. Biotechnology in Agriculture and Forestry, Medicinal and Aromatic plants VIII. Berlin: Springer-Verlag. p 36-46. 